ORIENTAL JOURNAL OF
COMPUTER SCIENCE \& TECHNOLOGY $\begin{array}{r}\text { ISSN: 0974-6471 } \\ \text { December 2016, } \\ \text { Vol. 9, No. (3): } \\ \text { Pgs. 194-203 }\end{array}$

\title{
Analysis of the Effect of Cell Phone Radiation on the Human Brain Using Electroencephalogram
}

\author{
HUMAIRA NISAR ${ }^{\star}$ and HAMARA M. AWADH \\ Department of Electronic Engineering Faculty of Engineering and Green \\ Technology Universiti Tunku Abdul Rahman, Malaysia. \\ *Corresponding author E-mail: humaira@ utar.edu.my \\ http://dx.doi.org/10.13005/ojcst/09.03.05
}

Received: March 16, 2016; Accepted: May 20, 2016)

\begin{abstract}
This paper aims to investigate the effect of cell phone radiation on human brain. It is known that the cell phone emits electromagnetic (EM) radiation which could be harmful to the human brain. In this research the electroencephalogram (EEG) signal has been acquired from 24 healthy subjects using a 16 channel EEG headset under different experimental conditions. The signal is decomposed into different brain rhythms using Daubechies Discrete Wavelet Transform up to 5th-level of the decomposition. Quantitative analysis has been carried out using two statistical parameters (Energy, Entropy) and Absolute Power. Special attention was focused on Temporal and Frontal lobes as these are near to the ear. Experimental results show higher values (for energy, entropy and absolute power) in the low-frequency bands (delta and theta) compared to the high frequency bands (alpha, beta and gamma) in both lobes. When the phone was placed $5 \mathrm{~cm}$ away from the head there was less brain activation compared to when the cell phone was placed next to the ear/head on both sides. It was found that there was more effect on the right side compared to the left side from the cell phone's radio waves.
\end{abstract}

Keywords: EEG; activation; Radio frequency; cell phone, Wavelet Transform, Entropy.

\section{INTRODUCTION}

The rapid evolution of the cell phone technology has resulted in a drastic increase in cell phone usage globally with an estimated 4.77 billion subscribers in 2017. The radio frequency (RF) used for communication by cell phone can penetrate through semi-solid substances, like living tissue to an effective distance proportional to its power density. According to Adey ${ }^{1}$, biophysical and physical studies demonstrate that sensitivity to very low RF fields have no direct or indirect relationship to cell or tissue heating. In 1999 Peyman and Chen $^{2}$ argued that if there are any adverse effects on the human brain, then it may be due to the nonthermal effect rather than the thermal effect. Recent research has demonstrated the short term effect of EM waves on the human brain, however, there is 
little or no evidence of the direct or indirect long term effect. Corle et al argue in [3] that although there is an increase in the number of reported brain tumors in the US, the cause is yet to be determined. Despite all this, scientists still believe that prolonged usage of cellular phone has potential adverse biological effects on the human health. Based on current statistics, mostly teenagers and children are the main victims of EM radiation. Children exposed to $\mathrm{RF}$ radiation at $1800 \mathrm{MHz}$ may have complications in the hypothalamus, cortical, hippocampus region and the eyes. Further studies have found that EM radiation penetrates deeper in a 5 year-old child's scalp than a 10 year-old and the lowest in an adult ${ }^{3}$.

Many studies have been carried out to understand or identify the risk of brain cancer from cell phone use. According to Morgan ${ }^{4}$, a large portion of the cost of most of the ongoing studies is paid by the cell phone industry itself, hence there is a possibility of poor study designs to favor the cell phone industry's image. In another study conducted by Kwon, et al., 13 healthy young males (aged 21 to 29 years and right-handed) $s$ were ON and OFF exposed to a cellular phone (Nokia 6110 Espoo, 902.4 MHz GSM) radio frequency waves to the right side of the head for a period of 30 minutes $^{5}$. During the experiment, subjects were asked to perform a simple visual task of identifying the $2 \mathrm{~cm}$-diameter circles of four different colors displayed on a monitor screen placed $1 \mathrm{~m}$ away for 0.5 seconds in a random order at random intervals of 2 to 5 seconds. Kwon, et al. (2011) concluded that, there was effect of the short-term GSM radiation on brain glucose metabolism in anterior and posterior parts of the right temporal cortex. In another experiment carried out by Maby, et al., two categories of 9 healthy and 6 epileptic patients were exposed to GSM mobile phone radiation ${ }^{6}$. He found that there was a change in alpha brain wave activity in both healthy and epileptic patients during the GSM mobile phone radiation exposure. In a study conducted by Tingolfa ${ }^{7}, 24$ healthy participants were exposed to cell phone radiation. It was found that there the brain electrical activity increases with the decrease in distance of the cell phone from the head. Tingolfa also found that the level of interference of the brain activity from the cell phone radiation is more on the right side.

In the following paragraphs we will give a brief overview of EM radiation, brain anatomy and EEG to understand this investigation. Cell phones transmit low RF power with operational frequencies in the range $450-2700 \mathrm{MHz}$ and peak powers of about 1-2 Watts. The peak power is highest around the cell phone's antenna and decreases with increasing distance. It is also agreed that, cell phone usage in an area with good reception reduces exposure. In bad reception area, the cell phone transmits high power to stay connected with the nearest base tower to sustain a call. Therefore, more RF energy is emitted hence an increase in the amount of exposure is expected.

The brain is one of the most important organs of the body that coordinates all the activities. It is divided into three major parts namely: Cerebrum, Cerebellum, brain stem and diencephalon. Cerebrum is the largest part of the brain. It covers the diencephalon and is divided into right and left hemisphere longitudinally. Each hemisphere is subdivided into brain lobes, named as: Frontal lobe, Parietal lobe, Temporal lobe and Occipital lobe. Teach lobe has its individual role. [8]. Frontal lobe takes care of parts of speech and movement, reasoning and emotions. The Parietal lobe deals with perception of stimuli in connection with temperature, pain and touch. The Temporal lobe takes care of hearing and memory. Finally the Occipital lobe deals with human vision. The brain signals are classified into frequency bands or brain rhythms, these are delta band $(0.5-4 \mathrm{~Hz})$, theta band $(4-8 \mathrm{~Hz})$, alpha band $(8-13 \mathrm{~Hz})$, beta band $(13-30 \mathrm{~Hz})$ and gamma band $(30-42 \mathrm{~Hz})$.

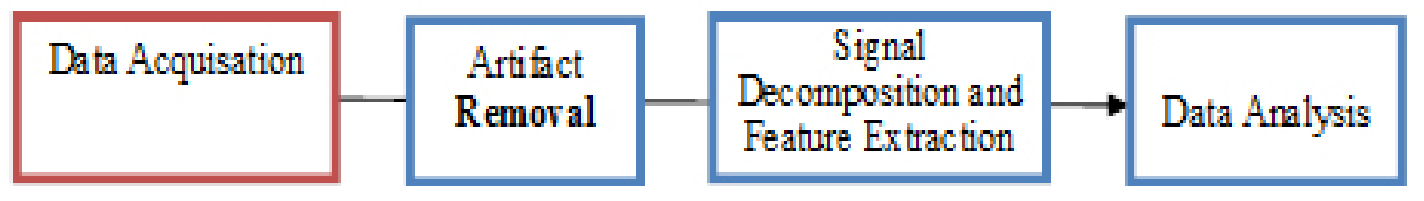

Fig. 1: Flow chart of the methodology 
The different brain bands represent different brain activities and characteristics. The communication within the brain is done by special messengers called neurons. EEG is the measure or recording of the brain's spontaneous electrical activity along the scalp. The brain consists of billions of neurons. These neurons fire randomly whenever a stimuli or a message is detected. The activities are represented as small electric voltage fields.

In order to carry out the analysis on the potential effect of cell phone usage on human brain, the brain rhythms of 24 healthy volunteers were recorded before, during and after cell phone usage for six distinct conditions. A quantitative analysis was carried out based on three statistical parameters (Entropy, and Standard Deviation) using Daubechies Discrete Wavelet Transform up to 5th-level of the decomposition in the frequency domain. Lastly, the Absolute Power was also calculated to correlate the results. The rest of the paper is organized as follows. In section III we will discuss the experimental setup for data acquisition. In section III, we will discuss the methodology for signal decomposition and quantitative analysis. In section IV, we will present experimental results and discussion on the results. Finally the paper will be concluded in section $\mathrm{V}$, followed by references.

\section{Experiment Setup for Data Acquisition}

Pre-recorded artifact free EEG data using 16-channel EEG head set from Emotiv Systems is used. Data is collected from 24 healthy participants. EEGLAB and Matrix Laboratory (MATLAB), is used for analysis. The data had been recorded for six conditions for 5 minutes each. After each recording the participants were given a 5 minute break. The experimental procedure is described as follows:

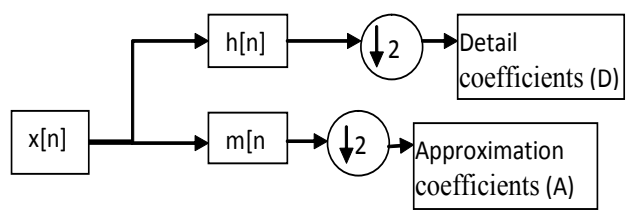

Fig. 2: Block diagram of a single level filter analysis
1. Before cell phone use: In relaxed mood with eyes open $(A)$

2. With cell phone: Left ear with no gap (B)

3. With cell phone: Left ear with a $5 \mathrm{~cm}$ gap (C)

4. With cell phone: Right ear with no gap (D)

5. With cell phone: Right ear with a $5 \mathrm{~cm}$ gap (E)

6. After cell phone use: In relaxed mood with eyes open $(F)$

\section{METHODOLOGY}

The project flow is as shown in Figure 1 below. The EEG data is acquired from 24 healthy participants. The data is processed by eliminating any artifacts present in the data manually and by using Independent Component Analysis (ICA) using EEGLAB. The artifact-free data is then analyzed in the frequency domain using Wavelet Transform using MATLAB environment.

EEG signal is decomposed using wavelet transform into different frequency bands. Wavelet transform can be categorized into three main categories; Continuous Wavelet Transform (CWT), Discrete Wavelet Transform and the Wavelet Packet. In DWT, a signal is sent through a string of filters. The signal is first sent through a pair of highpass and low-pass filters where convolution and decomposition are performed simultaneously. The output from the low-pass filter (Xlow) is called the approximation coefficient while the output from the high-pass filter (Xhigh) is called the detail coefficient as shown in Equations (1) and (2).

$$
X_{\text {high }}[n]=\sum_{t=-\infty}^{\infty} x[t] h[2 n-t]
$$

$$
X_{\text {low }}[n]=\sum_{t=-\infty}^{\infty} x[t] m[2 n-t]
$$

Both Xlow and Xhigh outputs contain only half of the original signal's frequencies since their time resolution is halved. This makes both outputs not able to meet the Nyquist criteria (fmax $>=f s / 2$ ). In order to make them meet the Nyquist rule, Xlow and Xhigh decimated or sub-sampled by a factor of 
two. This is shown in Fig. 2 for a single level wavelet analysis. For a multi-level wavelet analysis, the same procedure is repeated $\mathrm{N}$ (number of levels) times generating $\mathrm{N}$ detailed coefficients $(\mathrm{D})$ and $\mathrm{N}$ approximation coefficients $(\mathrm{A})$.

EEG signals are non-stationary signals, which means different frequency components are only present for unknown time periods in a given brain signal. Fourier Transform cannot describe it fully since it only involves stationary sinusoids. Wavelet transform [9] is a strong analytical tool for the non-Stationary signal analysis. It breaks down the EEG signal into different brain frequency bands (delta, theta, alpha, beta and gamma). In this work we have chosen the Daubechies Discrete Wavelet Transform (DWT, db2). The Daubechies is one of the most commonly used wavelet family by most researchers in EEG analysis. In this work we choose 5-level DWT (db2 and levels, l=5) for the analysis. To determine the number of levels required in a wavelet analysis for a certain signal, the sampling frequency of that signal is considered. This is because the splitting point of the decomposition is usually half the signals sampling frequency ( $\mathrm{fs} / 2$ or Nyquist frequency). In the current study, our sampling frequency is fs $=128 \mathrm{~Hz}$.

Two statistical parameters, Energy and Entropy were computed for the EEG signal frequency bands (gamma, beta, alpha, theta and delta). The parameters were calculated for all lobes. Energy is used to determine the energy distribution in an EEG signal. It explains the signal strength since it gives the area under the curve of power at all intervals of time. An increase in signal energy indicates the increase in brain activation. The EEG signal energy is given by Equation (3):

$$
\operatorname{Energy}(E(n))=\sum_{i=1}^{N}|D n i|^{2}, n=1,2,3
$$

Entropy is a measure of irregularity in signal. EEG signals are regular when there are less brain activities and may be irregular in the presence of radiation from mobile phone. The entropy of EEG signal is given by Equation (4):
$\operatorname{Entropy}(E N(n))=\sum_{i=1}^{N} D_{n i}^{2} \log \left|\left(D_{n i}^{2}\right)\right|, n=1,2,3$

where $D$ is an EEG detailed signal produced by wavelet transform.

\section{RESULTS AND DISCUSSION}

In this section we will discuss the change in brain activation observed using two statistical features (Energy and Entropy) and Power in different brain rhythms across the frontal and temporal lobes for six conditions for every subject.

The total energy for each condition in each frequency band and in each lobe (frontal and temporal) was calculated. The average total energy of the each brain frequency band in the temporal and frontal lobes is plotted for the six conditions In Figures 3 and 4. In general higher total energy values were recorded in the right ear than the left ear in all bands and in both lobes except in delta and theta band in the temporal region where left temporal lobe has higher activation for the case without distance. Higher total energy values were recorded when the cell phone was placed with the ear (without distance) during phone use compared to when the cell phone was placed at a distance from the ear $(5 \mathrm{~cm}$ away from ear) in all wave bands in both lobes.

Entropy is a measure of irregularity in an EEG signal. The Entropy value for each condition in each frequency band in each lobe (frontal and temporal) was calculated. The normalized entropy, in relation to condition 1 (before exposure) is shown in Figures 5 and 6 . In general higher total entrpoy values were recorded in the right ear than the left ear in all bands and in both lobes except in delta and theta band in the temporal region where left temporal lobe has higher activation for the case without distance. Higher total entropy values were recorded when the cell phone was placed with the ear (without distance) during phone use compared to when the cell phone was placed at a distance from the ear $(5 \mathrm{~cm}$ away from ear) in all wave bands in both lobes. 

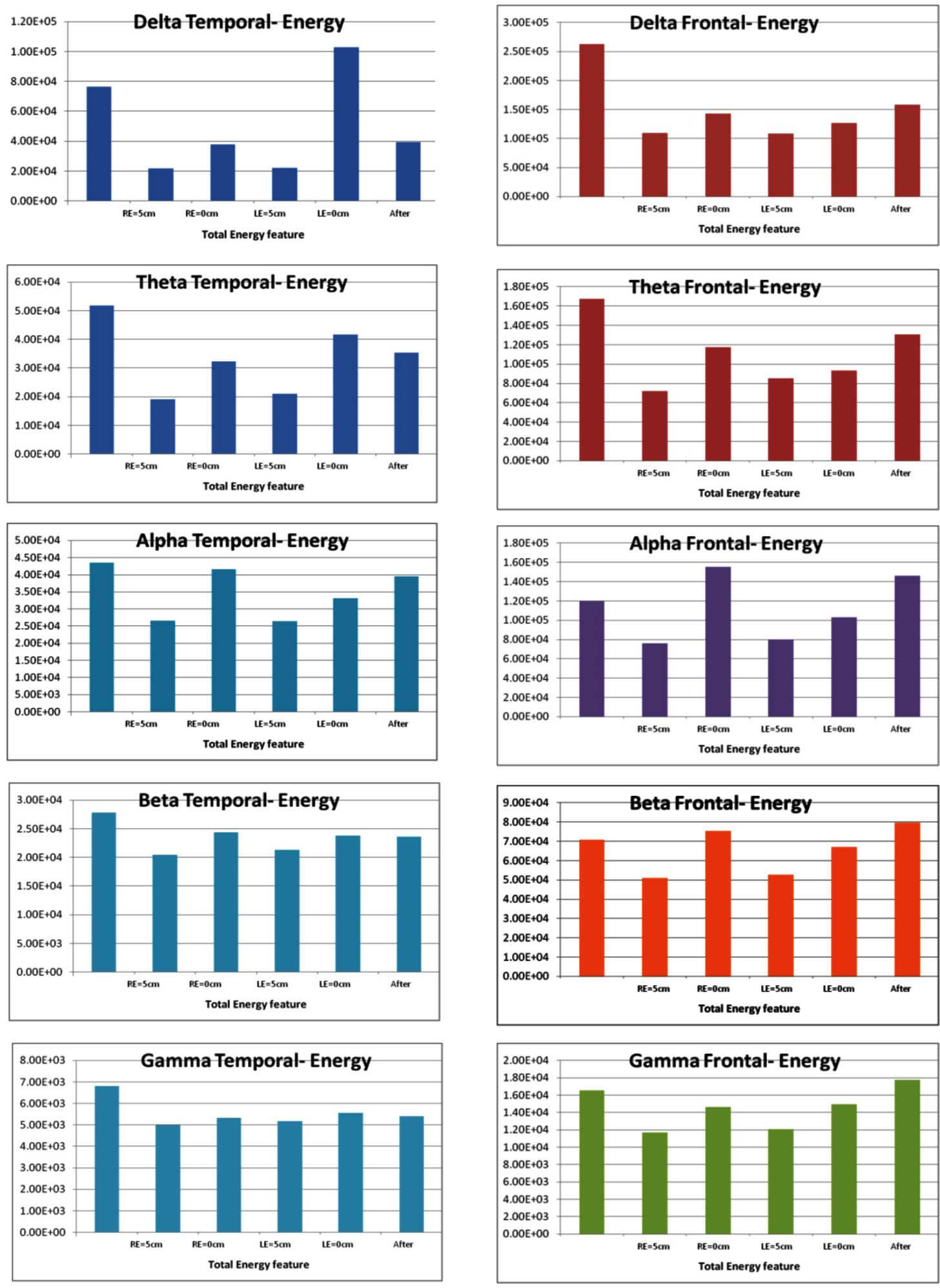

Fig. 3 Results of Energy in Temporal Lobe for different brain rhythms

Fig. 4: Results of Energy in Frontal Lobe for different brain rhythms 

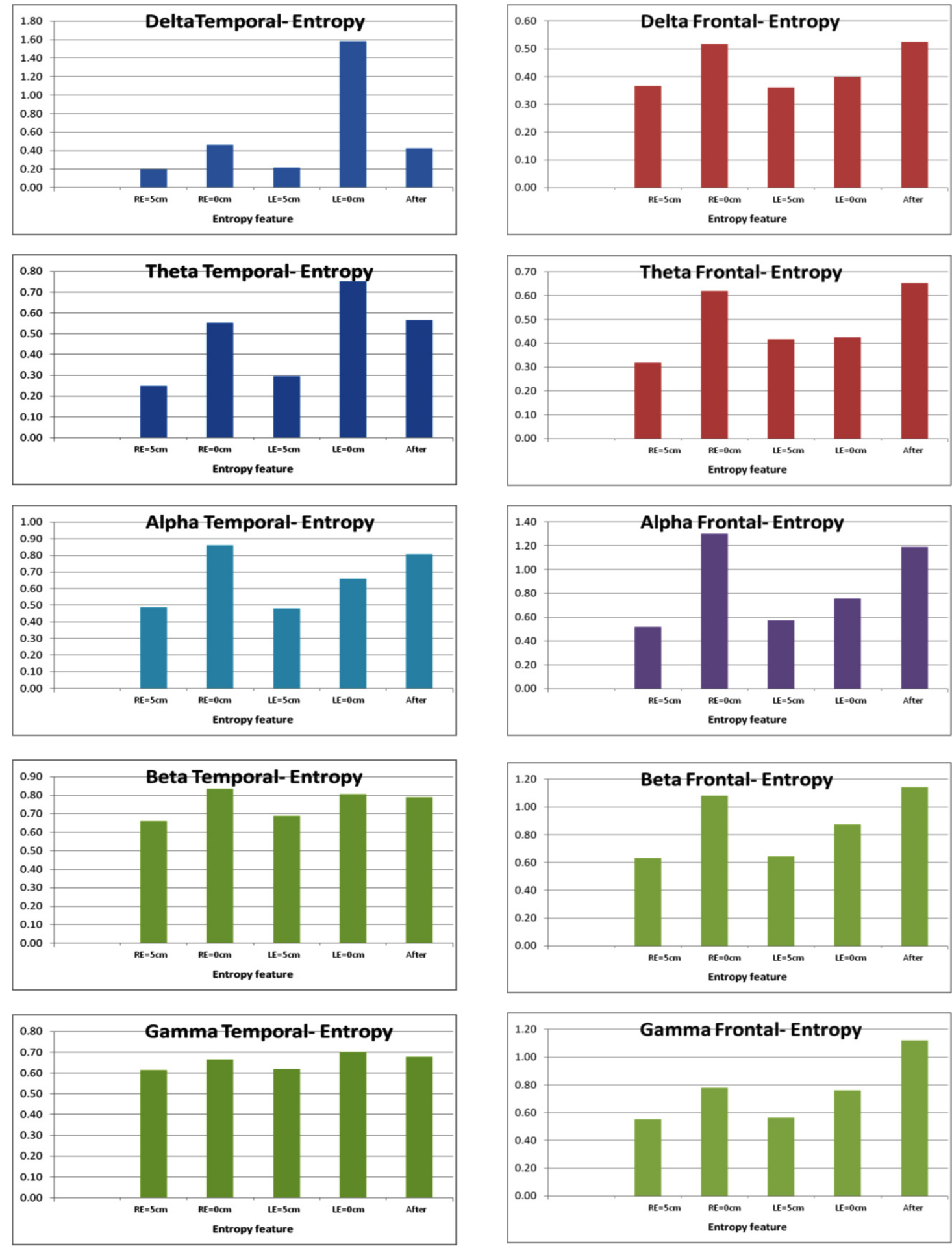

Fig. 5: Results of Entropy in Temporal Lobe for different brain rhythms

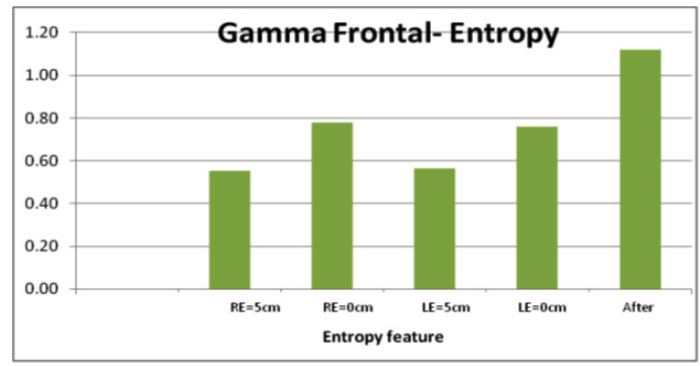

Fig. 6: Results of Entropy in Frontal Lobe for different brain rhythms 

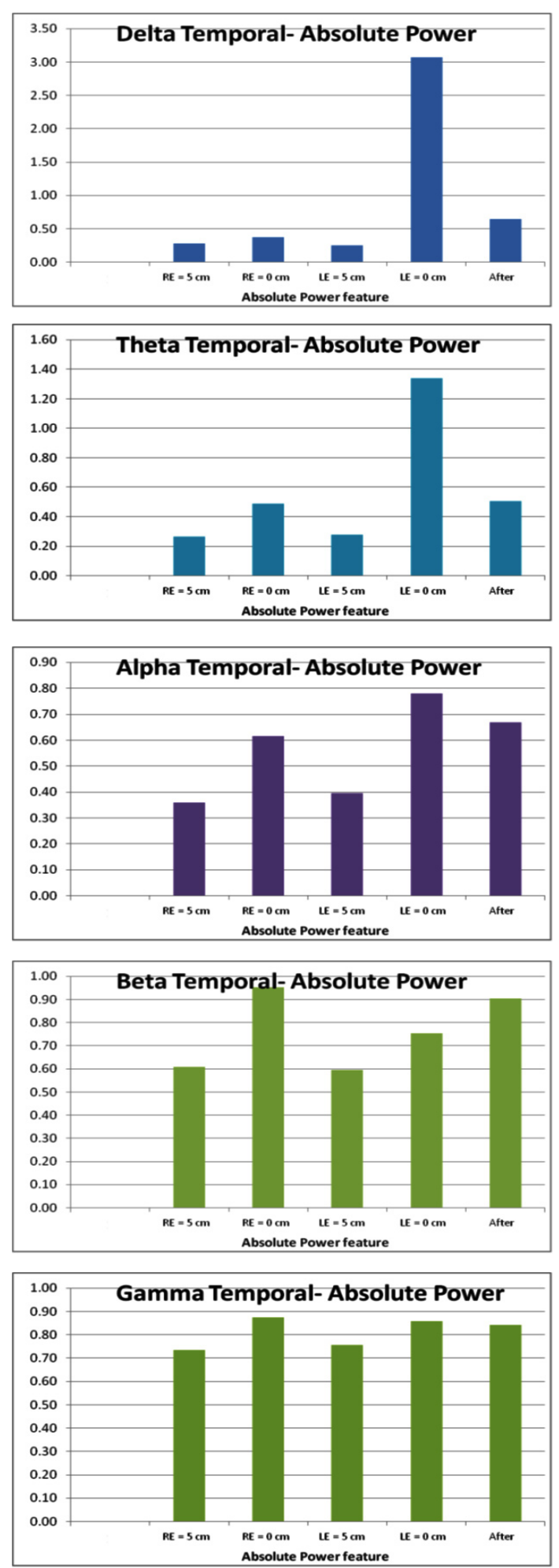

Fig. 7: Results of Absolute Power in Temporal Lobe for different brain rhythms
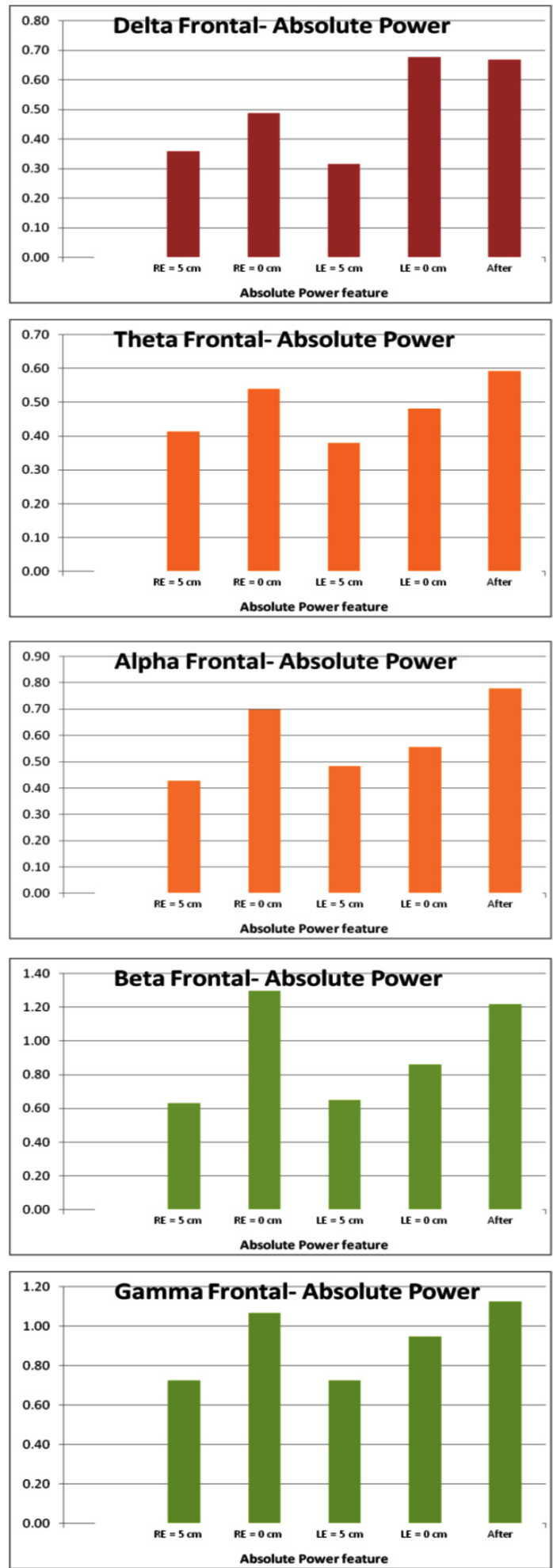

Fig. 8: Results of Absolute Power in Frontal Lobe for different brain rhythms 
A significant increase in signal entropy after cell phone use (the 6thCondition) was also recorded in theta, alpha, beta and gamma bands in both temporal and frontal lobes (Figure 5 and 6). This may suggest that the effect of cell phone radiation on these brain rhythms can last for some time even after exposure is over. Zandi, et al., reported that there is a fixed latency of about 15 minutes from the time of exposure to the time of observational changes in the EEG activities ${ }^{10}$. This may also explain the peak activity-values observed in the 6th Condition (after exposure) where EEG readings were recorded 5 minutes after the subject put down the phone.

Power is defined as the amount of total signal energy per unit time. The absolute power is generally expressed relative to baseline power level. In this work, the frequency-band powers, in relation to 1st Condition (before exposure) as the reference, were computed and expressed as the absolute power ratios of the EEG signals. The power ratios are plotted shown in Figure 7 and 8.

Higher absolute power values are recorded in the right ear than the left ear in all bands and in both lobes, except delta-Temporal, thetaTemporal and alpha-Temporal and delta-Frontal bands. Higher absolute power values were recorded when the cell phone was placed next to the the ear (without distance) during phone use compared to when the cell phone was placed at a distance from the ear ( $5 \mathrm{~cm}$ away from ear) in all bands and in both lobes. The absolute power findings are almost inline with our findings with Energy and Entropy.

In this work we have found that higher feature values (energy, entropy and absolute power) have been recorded in the low-frequency bands (delta and theta) compared to the high frequency bands (alpha,beta and gamma) in both lobes. Overall, the delta band has shown the highest values in this study. Delta waves are the most basic in a relaxed person's brain signal and they carry the most signal energy due to their high amplitudes and slow-frequency. Several studies of brain waves have reported that delta and theta waves increase with sleep in normal human adults and decrease in a wakeful brain state. However, in this study we found there were increase in delta and theta wave energy and entropy during cell phone use (during a wakeful brain state). Since the high increase in energy and entropy values in the low frequency bands indicates the presence of delta and theta waves in large percentage in a wakeful state, this primarily suggests an interference of the brain normal activity by the cell phone's radio waves which are emitted closely to the head during ordinary cell phone use.

In this study, it has also been found that cell phone use with a distance of $5 \mathrm{~cm}$ (in the current study) has less effect compared to without a distance at both the left and right ears. When the phone was placed $5 \mathrm{~cm}$ away from the head there were less brain activities compared to more activities when the cell phone was placed right against the ear in both sides. These findings indicate that a cell phone's RF radiation is highest around the cell phone's antenna and decreases with increasing distance. Hence, the amount of induced brain activity decreases with distance during phone use.

In this study the right ear without distance has shown a typical dominant and consistent trend of having the highest signal energy and entropy values followed by the left ear without distance. However, with the exception of delta-Temporal and theta-Temporal which have highest values at the left ear without distance. Sekimoto, et al. argue that in a normal adult's brain at rest, delta waves have a higher count of dominance in the righthemisphere (frontal and central lobes) than in the left-hemisphere [11]. This high dominance of delta waves in the right-hemisphere compared to the lefthemisphere supports our findings of the right-ear without distance showing the highest brain activity (delta-Frontal) during cell phone use.

A higher dominance of delta waves in the left-hemisphere (temporal) mainly suggests a brain confusion or a serious brain disorder like schizophrenia. However this is a very preliminary study and we cannot conclude that what is the exact reason for this observation. However it may be due to the brain's exposure to cell phone's radio waves that leads to the abnormal sharp spike of delta-Temporal in the left-hemisphere. 
The presence of large counts of delta and theta waves in a wakeful state (as found in this study) is just abnormal and may result in decrease in brain concentration or may lead to brain confusion. Hence, this also supports the various reports from previous studies of anxiety and headaches after a phone call. It is also important to note that the low-frequency radio waves emitted by the cell phone are more likely to interfere with the low-frequency components of a brain signal rather than with the high-frequency components. This may also explain why there are significant amount of activities in the delta band compared to the rest of the bands.

Higher energy values were recorded before exposure because the subjects were more excited at the beginning of the experiment but with time they got used to experimenting environment and were able to achieve a relaxed brain state. Based on this study, significant changes in entropy and energy values were recorded in the alpha band. Alpha waves are the dominant wave patterns of a normal adult in a wakeful and relaxed state with eyes closed. Typically, alpha waves decrease in amplitude when eyes opened. Therefore, the findings in this study of increased alpha energy, entropy and standard deviation during cell phone use (eyes opened) clearly indicates the presence of abnormal brain activities induced or triggered in the alpha band during the process.

\section{CONCLUSION}

An analysis for effect of cell phone radiation on the human brain was carried out in the frequency domain. The EEG signal was decomposed into various frequency bands by using Daubechies Discrete Wavelet Transform. In the feature extraction, entropy, and energy of the transformed signals were calculated and their average values plotted for temporal and frontal lobes. These are the lobes nearest to the ear and hence get most exposure during cell phone use. It was found that higher signal energy and entropy values were recorded during cell phone use without distance than with a distance of $5 \mathrm{~cm}$. Hence there is more risk of radiation effect on the brain when the cell phone is placed next to the ear than when holding it at a distance from the ear. This is because a cell phone's RF radiation is highest around the cell phone's antenna and decreases with increasing distance.

Typical alpha waves decrease in amplitude with eyes opened and increase when eyes closed and at a relaxed brain state. Contrary to that, in this study, there were increase in alpha energy and entropy with eyes open. Therefore, this clearly indicates the presence of brain activities induced or triggered in the alpha band during cell phone use. The delta energy and entropy are higher in temporal lobe (delta-temporal) at left-ear without distance than at right-ear without distance in this study. In this work, low-frequency bands (delta, theta and alpha) have consistently recorded highest values in most cases compared to the high-frequency bands (beta and gamma). Additionally, from all the findings, it can be concluded that delta, theta and alpha bands have more effect from the cell phone radiation than the rest of the bands.

In conclusion we can say that there is slightly higher effect on the right-ear compared to the left-ear from the cell phone's radio waves. This may be due to the high dominant delta waves in the right-hemisphere (frontal and central lobes) than in the left-hemisphere as argued by Sekimoto, et $\mathrm{al}^{10}$. Also this is why the frontal lobe recorded higher overall delta energy and entropy values compared to the corresponding delta band in the temporal lobe with only one spike of peak values at the left-ear without distance.

\section{REFERENCES}

1. Adey, W. Interaction Mechanism of Low Level Electromagnetic Fields in Living Systems. Collective Properties of Cell Membrane, pp. 47-77, (1977).
2. Peyman, A. \& Chen, X. Electromagnetic Assessment and Antenna Design Relating to Health Implication of Mobile Phone. The feasibility of using medical imaging modalities 
for in-vivo detecting of physiological effects of electromagnetic radiation in the human brain during the use of cellular phones, pp. 3/1-3/6 (1999).

3. Corle, C., Makale, M. \& Kesari, S. Cell phones and glioma risk: a review of the evidence. 106(1), pp. 1-13 (2012).

4. Morgan, L. Powerwatch. [Online] Available at:http://www.powerwatch.org.uk/columns/ morgan/20080108_interphone_design. asp[Accessed 11 July 2014] (2014).

5. Kwon, M. S. et al. GSM mobile phone radiation suppresses brain glucose metabolism. Journal of Cerebral Blood Flow \& Metabolism, 31(12), p. 2293-2301 (2011).

6. Maby, E., es, R. L. B. J. \& Faucon, G. Short-term effects of GSM mobiles phones on spectral components of the human electroencephalogram.New York City, IEEE (2006).
7. Tingolfa, T. Analysis of the Side Effects of Cell Phone on the Brain Using EEG, Tronor, Perak: Universiti Tecknologi PETRONAS (2013).

8. Humaira Nisar, Yeap Kim Ho, "Introduction to EEG and ERP Signals," EEG/ERP Analysis: Methods and Applications, Taylor and Francis K22384, (2014).

9. Panda, R. et al., . Classification of EEG signal using wavelet transform and support vector machine for epileptic seizure diction. Systems in Medicine and Biology (ICSMB), pp. 405-408 (2010).

10. Zandi, S. et al. A Novel Wavelet Based Index to Detect Epileptic Seizures Using Scalp EEG Signals. 30th Annual International IEEE EMBS Conference Vancouve, British Columbia, Canada, 1 (2008).

11. Sekimoto, M. et al. Reduced Frontal Asymmetry of Delta Waves During All-Night Sleep in Schizophrenia. Schizophr Bull, 33(6), p. 1307-1311: (2006). 